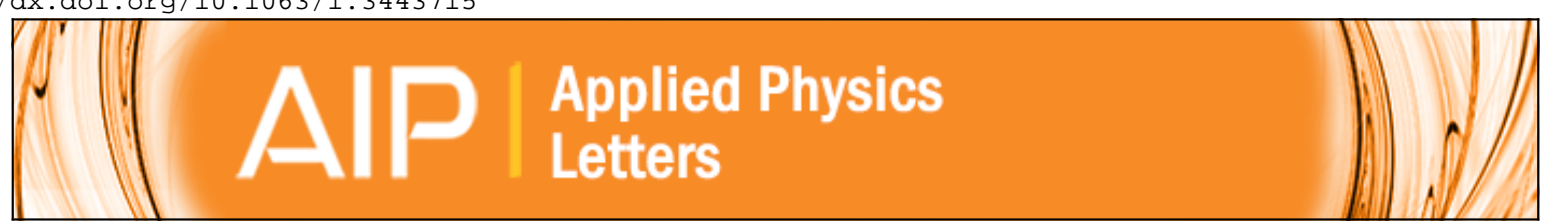

Control of magnetic and electric responses with electric and magnetic fields in magnetoelectric heterostructures

J. Das, M. Li, S. S. Kalarickal, S. Altmannshofer, K. S. Buchanan, J. F. Li, and D. Viehland

Citation: Applied Physics Letters 96, 222508 (2010); doi: 10.1063/1.3443715

View online: http://dx.doi.org/10.1063/1.3443715

View Table of Contents: http://scitation.aip.org/content/aip/journal/apl/96/22?ver=pdfcov

Published by the AIP Publishing

Over 700 papers \&
presentations on

multiphysics simulation wew now

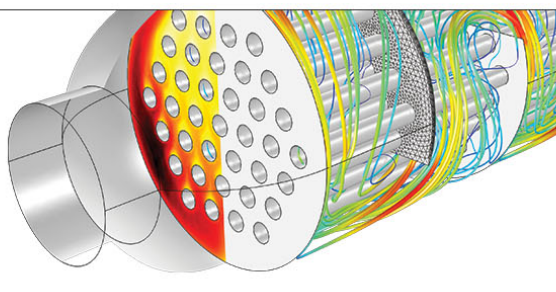




\title{
Control of magnetic and electric responses with electric and magnetic fields in magnetoelectric heterostructures
}

\author{
J. Das, ${ }^{1, a)}$ M. Li, ${ }^{1}$ S. S. Kalarickal, ${ }^{2,3}$ S. Altmannshofer, ${ }^{3}$ K. S. Buchanan, ${ }^{3}$ J. F. Li, ${ }^{1}$ and \\ D. Viehland ${ }^{1}$ \\ ${ }^{1}$ Department of Materials Science and Engineering, Virginia Tech, Blacksburg, Virginia 24061, USA \\ ${ }^{2}$ Center for Magnetism and Magnetic Nanostructures, University of Colorado, Colorado Springs, \\ Colorado 80933, USA \\ ${ }^{3}$ Department of Physics, Colorado State University, Fort Collins, Colorado 80523, USA
}

(Received 16 April 2010; accepted 12 May 2010; published online 4 June 2010)

\begin{abstract}
This paper reports on the tuning of both magnetic and electric responses with electric and magnetic fields for metglas- $\mathrm{Pb}(\mathrm{Zr}, \mathrm{Ti}) \mathrm{O}_{3}$ based magnetoelectric $(\mathrm{ME})$ heterostructures that can be promising for communication and sensor applications. The hysteresis loop results indicate a change in the in-plane magnetization due to application of voltages that leads to a tuning of the ferromagnetic resonance frequency by up to about $210 \mathrm{MHz}$ with electric field. Furthermore, these structures show a high ME voltage coefficient that results in the detection of a $2 \mathrm{nT}$ ac magnetic field and a low noise floor. (C) 2010 American Institute of Physics. [doi:10.1063/1.3443715]
\end{abstract}

Tuning of the magnetic response with electric field, and of the electric response with magnetic field, in magnetoelectric (ME) heterostructures have drawn considerable attention in the past several years ${ }^{1,2}$ due to their potential for cost effective and fast communication and sensor device applications. Such ME structures contain magnetic and electric materials that are in close contact with each other. It has been shown that coupling between the magnetic and electric phases can offer control of the magnetic/electric responses under electric/magnetic field, ${ }^{3,4}$ for example, through stress tuning. High ME coupling between constituent magnetostrictive and and piezoelectric phases in the stress tuned structures allows the possibility of achieving both types of cross-tuning: control of magnetism via an electric field, and control of electric polarization through the application of a magnetic field.

The potential to control the magnetic response with electric field at microwave frequencies in the ME structures would allow one to tune the ferromagnetic resonance (FMR) frequencies simply by applying a bias voltage in the 5-12 $\mathrm{GHz}$ frequency range. A number of heterostructures made of various magnetic (for example, $\mathrm{Fe}_{3} \mathrm{O}_{4}$ ) and piezoelectric (for example, $\mathrm{Pb}(\mathrm{Mg}, \mathrm{Nb}) \mathrm{O}_{3}-\mathrm{PbTiO}_{3}$ or $\left.\mathrm{PMN}-\mathrm{PT}\right)$ materials have been studied to date. ${ }^{3,5}$ Further, the provision to control the electric response with a magnetic field would allow one to use these ME structures as magnetic field sensors with sensitivities in the nano-Tesla (nT) range or higher that are expected to be much cheaper than the currently available sensors (for example, fluxgate). Combinations of various magnetic materials (for example, metglas) and piezoelectric phases (for example, $\mathrm{Pb}(\mathrm{Zr}, \mathrm{Ti}) \mathrm{O}_{3}$ or $\mathrm{PZT}$ ), and of various relative orientations of magnetization and polarization have been studied to date. ${ }^{6,7}$ These promising recent developments have set the ground for further investigations on structures with different magnetic and electric materials that might be useful for 1) practical electric-field-controlled microwave device applications and 2) portable and cheap, highly sensitive ME sensor applications.

${ }^{a)}$ Electronic mail: jaydip@vt.edu.

Here, we demonstrate both types of cross-tuning properties: 1) control of the FMR response under electric field at $9.5 \mathrm{GHz}$ and 2) control of the polarization with magnetic field at $1 \mathrm{~Hz}$, for metglas-PZT based ME laminate structures with various optimized geometries. The FMR response for a bimorph structure is found to have a notable electric field tunability of up to $210 \mathrm{MHz}$ at $9.5 \mathrm{GHz}$ due to a change of the effective in-plane magnetic induction with applied voltage. Structure based on push-pull configuration shows a high ME response under applied magnetic field and an ac magnetic field sensitivity of $2 \mathrm{nT}$ at $1 \mathrm{~Hz}$.

Figure 1 shows schematic of the various geometric configurations for the metglas and PZT layers that we have studied. Parts (a) and (b) show unimorph and bimorph structures, respectively. The unimorph structure contains one metglas layer $\left(0.025 \times 4 \times 5 \mathrm{~mm}^{3}\right)$ and one PZT layer $(0.2 \times 4$ $\times 5 \mathrm{~mm}^{3}$ ) along with interdigitated (ID) electrodes, as indicated. The bimorph is a sandwiched PZT-metglas-PZT structure of similar respective layer dimensions as the unimorph structure. These configurations can result in longitudinal orientations for both the magnetization $(M)$ and polarization $(P)$, which are expected to offer a better ME coupling between layers. ${ }^{7}$ Further, the bimorph configuration should result in a higher effective interface-driven strain because for this structure, the metglas volume in contact with PZT is

(a) Unimorph

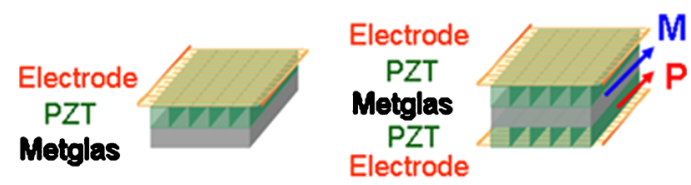

(c) Push-pull

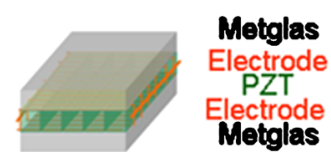

FIG. 1. (Color online) Schematics of the (a) unimorph, (b) bimorph, and (c) push-pull configurations for the PZT-metglass structures along with the ID electrodes. The magnetization $(M)$ and polarization $(P)$ directions are as indicated. 
higher than that of the unimorph configuration. Thus, it should show a larger ME coefficient as well as a larger FMR frequency shift. Part (c) shows a schematic of the push-pull mode with the PZT $\left(0.2 \times 10 \times 10 \mathrm{~mm}^{3}\right)$ layer sandwiched in between two metglas $\left(0.075 \times 10 \times 10 \mathrm{~mm}^{3}\right)$ ones. For this configuration, the relative orientation of the $M$ and $P$ is known to have a high ME coefficient and a high magnetic field sensitivity. For all the structures, the PZT and metglas layers along with the ID electrodes were bonded together with epoxy resin following the similar preparation process as discussed in Ref. 4.

We then measured the change in the magnetic responses with electric fields using a vibrating sample magnetometer and a shorted waveguide FMR measurement technique for both the unimorph and bimorph structures. Magnetization hysteresis loops were obtained for in-plane static magnetic fields under various voltages applied across the PZT layer. The FMR experiments were done with an in-plane static magnetic field at frequencies ranging from 8 to $12 \mathrm{GHz}$ following the same procedure detailed in Ref. 8. The samples were placed at the center of the waveguide wall and were connected to a high voltage source with insulated copper wires to apply voltages through the ID electrodes across the piezoelectric layers. Commercial tapes were used to fix the sample and wires in the waveguide. Voltages of 0 to $1000 \mathrm{~V}$ were applied to study the electric field control of the FMR response.

Figure 2 shows the hysteresis loop results. Part (a) shows representative hysteresis loops for applied voltages of 0 and $1000 \mathrm{~V}$, as indicated. The inset shows an enlargement of the $M-H$ loops near saturation. One can see that the in-plane magnetic induction $4 \pi M$ is decreased in the magnetic field range of about 100-380 Oe when the electric voltage is applied. Under higher magnetic fields, the $4 \pi M$ remains unaffected. Parts (b) through (d) show the changes in the $4 \pi M$ as a function of the applied voltages at magnetic fields of $H$ $=50,150$, and 350 Oe for both the unimorph and bimorph structures, as indicated. For $H=50$ Oe, the changes in $4 \pi M$ are nonlinear with applied voltage. The bimorph structure shows nearly double the change in $4 \pi M$ relative to that for the unimorph structure. For higher magnetic fields, the change in $4 \pi M$ with electric field is smaller and nearly linear for both the structures. For $H=350$ Oe, the $4 \pi M$ does not change with voltage for the unimorph structure, whereas the bimorph structure still exhibits some linear change.

Figure 3 shows the $9.5 \mathrm{GHz}$ FMR results. Parts (a) and (b) show the unimorph and bimorph FMR absorption derivative profiles as a function of static magnetic field for various applied voltages, as indicated. In Part (a), one can see a small 8 Oe shift in the FMR profile for the unimorph to the lower field side when a voltage of $1000 \mathrm{~V}$ is applied. Part (b) shows the FMR profiles for the bimorph for voltages of $-250,0$, and $1000 \mathrm{~V}$. We can see that under positive voltage, the FMR profile shifts to the lower field side by about $75 \mathrm{Oe}$, which is a much larger shift as compared to what is observed for the unimorph. Under negative voltages, the FMR profile shifts to the higher field side. Part (c) shows the shift in the FMR frequency, determined from the resonance field shift, as a function of applied electric field for the bimorph structure. One can see that the FMR frequency follows a linear behavior with applied voltage, as indicated by the solid line in the graph.
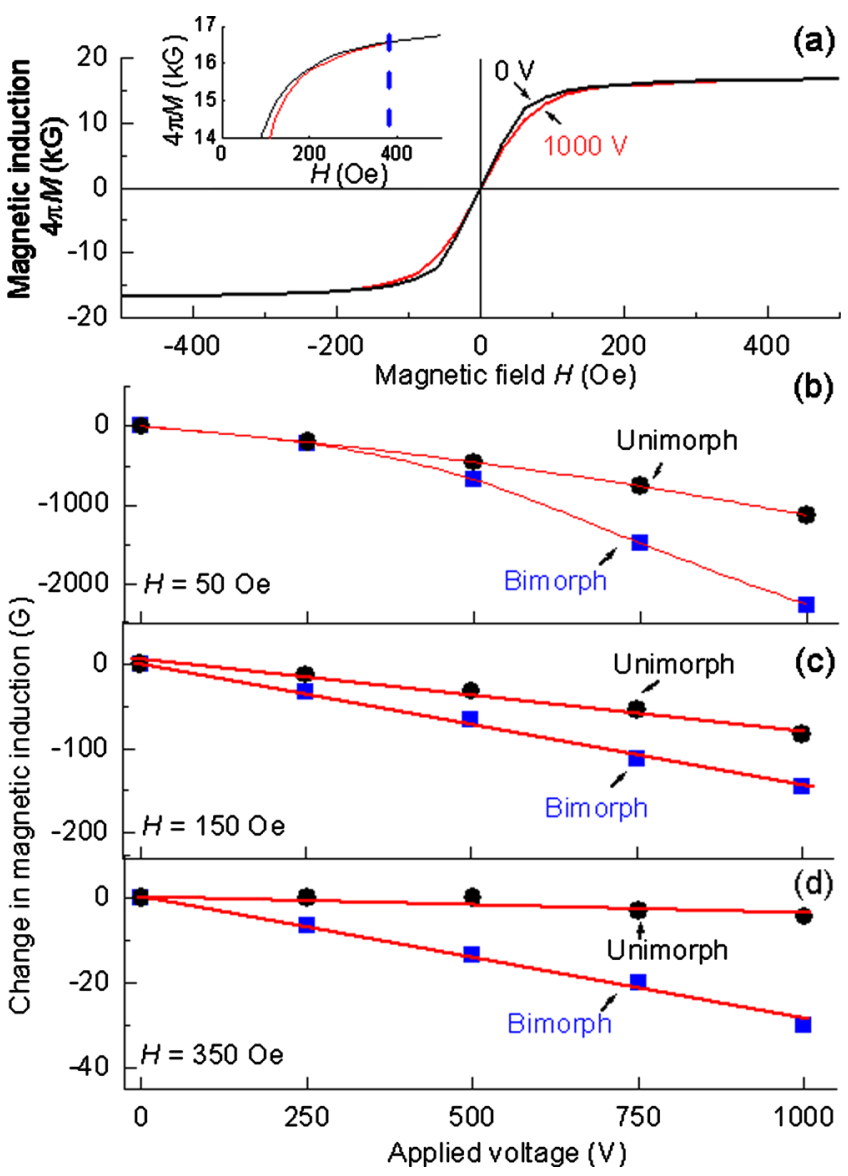

FIG. 2. (Color online) (a) Representative in-plane hysteresis loops for the heterostructure for 0 and $1000 \mathrm{~V}$ across the PZT layer. Inset shows a closer view of the field region where the magnetization differs. The change in in-plane magnetic induction as a function of the applied voltage at static magnetic field $H$ of (b) $50 \mathrm{Oe}$, (c) $150 \mathrm{Oe}$, and (d) $350 \mathrm{Oe}$ for the unimorph and bimorph structures, as indicated. The lines are drawn as a guide to eye.

For the in-plane magnetic field configuration, the resonance condition for the metglas-PZT structure can be written as,

$$
\omega=|\gamma| \sqrt{ }\left(H_{0}+H_{\text {eff }}\right)\left(H_{0}+4 \pi M_{\mathrm{s}}+H_{\text {eff }}\right),
$$

where $\omega$ is the resonance frequency, $|\gamma|=2.8 \mathrm{GHz} / \mathrm{kOe}$ is the gyromagnetic ratio, $H_{0}$ is the static magnetic field at resonance, and $4 \pi M_{\mathrm{s}}$ is the saturation induction. An effective magnetic field $H_{\text {eff }}$ designates the electric-field-induced anisotropy field that results in a modification of the in-plane magnetization. As one applies an electric field, the PZT layer expands and the piezoelectric coupling coefficient $d_{33}$ increases or decreases linearly with positive or negative electric field. ${ }^{9}$ This change in $d_{33}$ affects the adjacent magnetostrictive layer, yielding a proportional change in the $H_{\text {eff. }}$. The linear change in $4 \pi M_{\mathrm{s}}$ with applied voltage under higher magnetic fields (see Figs. 2(b) and 2(c)) and the linear shift in the resonance frequency with applied voltage (see Fig. $3(\mathrm{c}))$ can be attributed to the change in $H_{\text {eff }}$ of the metglas layer caused by electric-field-induced changes in $d_{33}$ of the PZT layer. For the bimorph structure, calculated value for the microwave ME coefficient $\alpha_{\text {ME-MICROWAVE }}$ from the resonance frequency shift data is about $20 \mathrm{MHz} \mathrm{cm} / \mathrm{kV}$. This is about one-fourth of the expected value following the estimations in Ref. 10 and 15 times lower than the highest value of $300 \mathrm{MHz} \mathrm{cm} / \mathrm{kV}$ reported so far for a $\mathrm{Fe}_{3} \mathrm{O}_{4}$-PMN-PT structure in Ref. 5 A 5 times smaller $d_{3}$ coefficient for the 

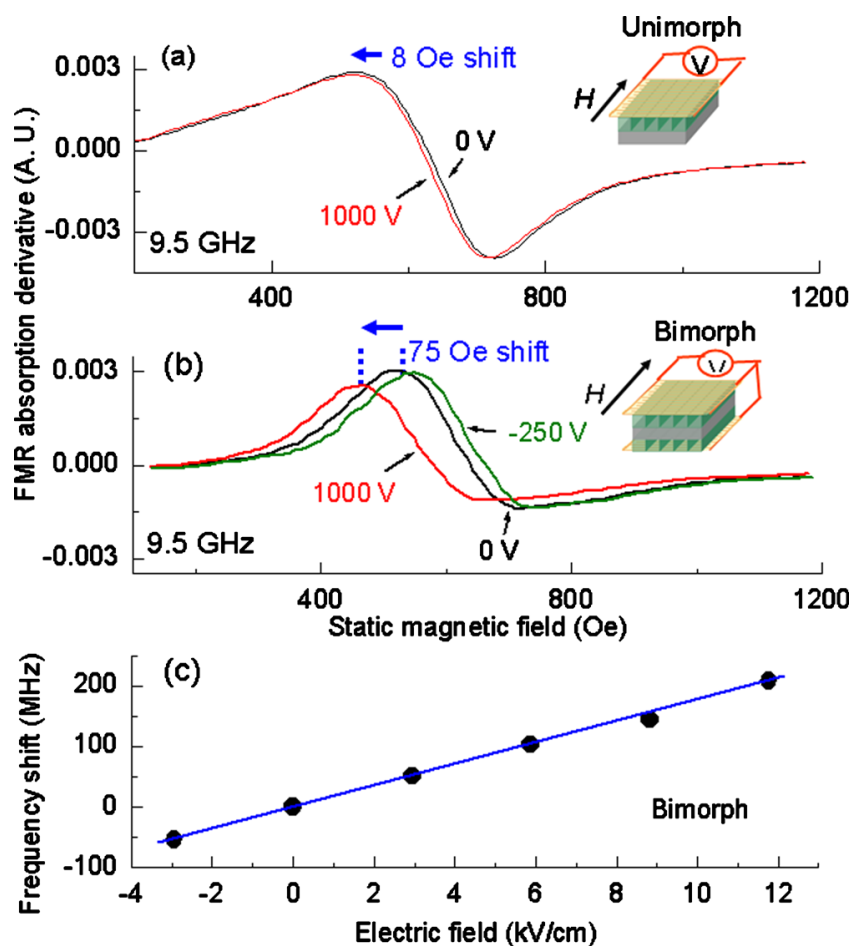

FIG. 3. (Color online) FMR absorption derivative as a function of the static magnetic fields for (a) unimorph and (b) bimorph structures at different applied voltages, as indicated. The schematics show the magnetic field and voltage configurations. (c) The shift in the FMR frequency as a function of the electric field for a bimorph structure. The solid line is drawn as a guide to eye.

PZT ceramics than the PMN-PT single crystals and the decrease in the metglas piezomagnetic coefficient in the resonance field range are possibly the reasons for lower $\alpha_{\text {ME-MICROWAVE }}$ obtained in this study.

We next characterized the magnetic field tuning of the electric response for the push-pull mode structure and the results are shown in Fig. 4.We measured the voltages induced across the ID electrodes using a lock-in amplifier as a function of dc magnetic field $H_{\mathrm{dc}}$ and in response to a Helmholtz coil driven ac field of $1 \mathrm{Oe}$ at a frequency of $1 \mathrm{kHz}$. Part (a) shows the ME voltage coefficient $\alpha_{\mathrm{ME}-\mathrm{V}}$ as a function of the static magnetic field. One can see that $\alpha_{\mathrm{ME}-\mathrm{V}}$ increases with increasing $H_{\mathrm{dc}}$; reaches a maximum point, and then drops of as $H_{\mathrm{dc}}$ is further increased. We than fixed $H_{\mathrm{dc}}$ at a point that was needed to obtain the highest $\alpha_{\mathrm{ME}-\mathrm{V}}$ and measured the sensitivity to small variations in ac magnetic field (in the order of nT). The calibration technique for such measurements has been previously detailed in Ref. 4. Part (b) shows the noise level and sensitivity results, as indicated. One can see an ac signal with a peak-to-peak value of about $8.1 \mathrm{mV}$, in response to a $2 \mathrm{nT}$ ac magnetic field at $1 \mathrm{~Hz}$, is needed to obtain a signal-to-noise ratio greater than 2 . Finally, we measured the noise floor in the $1-1000 \mathrm{~Hz}$ frequency range using an operational amplifier with a detection circuit of gain $1 \mathrm{pC} / \mathrm{V}$. Part (c) shows the noise power spectrum for this heterostructure. The dip at $60 \mathrm{~Hz}$ originates from the filter in our detection circuit. One can see that the noise floor is about $0.5 \mathrm{nT} / \mathrm{Hz}^{0.5}$ in the frequency range of $1-10 \mathrm{~Hz}$ and $0.6 \mathrm{nT} / \mathrm{Hz}^{0.5}$ in the range of $100-1000 \mathrm{~Hz}$. It should be noted that smaller heterostructures, as presented here, might serve better than the previously reported struc-
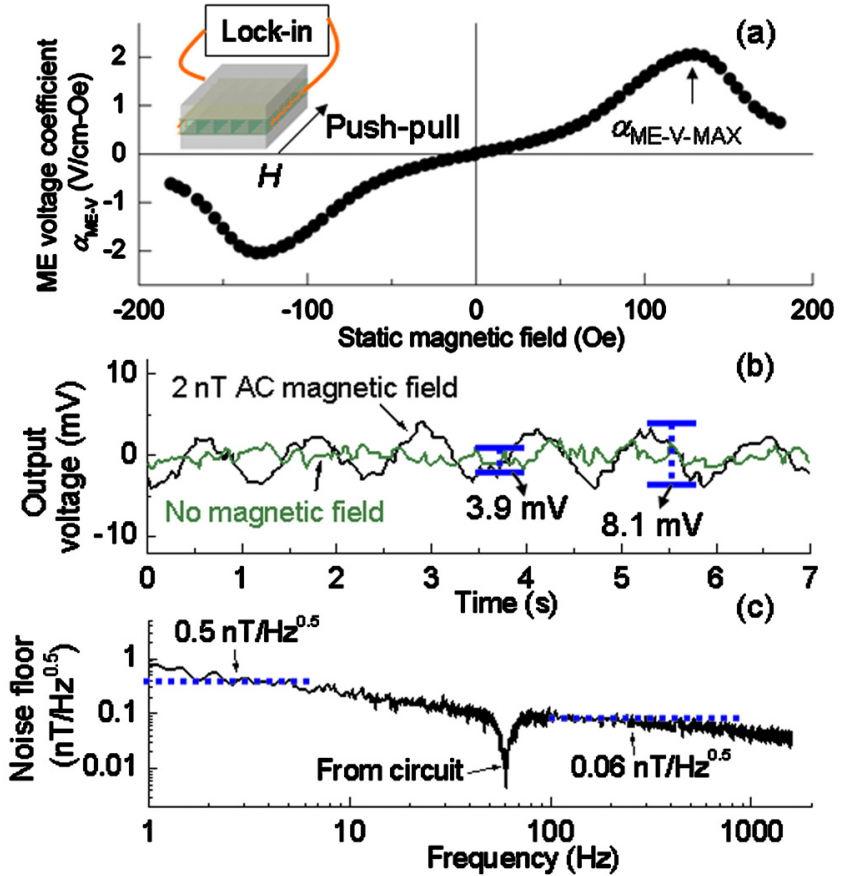

FIG. 4. (Color online) (a) The ME voltage coefficient $\alpha_{\mathrm{ME}-\mathrm{V}}$ as a function of the static magnetic field. The maximum response point has been indicated by an arrow. (b) Output signals as a function of time for zero and $2 \mathrm{nT}$ ac magnetic field at $1 \mathrm{~Hz}$. The peak-to-peak voltages are as indicated. (c) Noise floor response as a function of frequency. The dashed lines indicate the responses in the $1-10 \mathrm{~Hz}$ and $100-1000 \mathrm{~Hz}$ frequency range.

tures with larger dimensions ${ }^{4}$ for cheap and portable magnetic field sensor devices.

In summary, both electric field control of the magnetic response and magnetic field control of the electric response have been demonstrated for metglas-PZT based unimorph, bimorph, and push-pull configurations. The bimorph structure shows a better electric-field-controlled $\mathrm{ME}$ response than the unimorph structure. FMR results indicate a 210 $\mathrm{MHz}$ shift in the resonance frequency under applied electric field of about $12 \mathrm{kV} / \mathrm{cm}$. The push-pull configuration shows a high ME voltage coefficient, giving rise to a high sensitivity of about $2 \mathrm{nT}$ in response to an ac magnetic field at $1 \mathrm{~Hz}$ for these small heterostructures.

This work was supported in part by AFOSR under Grant No. FA 9550-09-1-0552 and ONR under Grant No. N0001410-1-0257, and NSF under Grant No. DMR-0907706.

${ }^{1}$ M. Fiebig, J. Phys. D 38, R123 (2005).

${ }^{2}$ C.-W. Nan, M. I. Bichurin, S. Dong, D. Viehland, and G. Srinivasan, J. Appl. Phys. 103, 031101 (2008).

${ }^{3}$ J. Das, Y. Song, N. Mo, P. Krivosik, and C. E. Patton, Adv. Mater. 21, 2045 (2009)

${ }^{4}$ J. Das, J. Gao, Z. Xing, J. F. Li, and D. Viehland, Appl. Phys. Lett. 95, 092501 (2009).

${ }^{5}$ M. Liu, O. Obi, J. Lou, Y. Chen, Z. Cai, S. Stoute, M. Espanol, M. Lew, X. Situ, K. S. Ziemer, V. G. Harris, and N. X. Sun, Adv. Funct. Mater. 19, 1826 (2009).

${ }^{6}$ S. X. Dong, J. F. Li, and D. Viehland, Appl. Phys. Lett. 83, 2265 (2003).

${ }^{7}$ J. Zhai, Z. Xing, S. X. Dong, J. F. Li, and D. Viehland, J. Am. Ceram. Soc. 91, 351 (2008).

${ }^{8}$ J. Das, S. S. Kalarickal, K. Kim, and C. E. Patton, Phys. Rev. B 75, 094435 (2007).

${ }^{9}$ Y. K. Fetisov and G. Srinivasan, Appl. Phys. Lett. 93, 033508 (2008),

${ }^{10}$ G. Srinivasan, I. V. Zavislyak, and A. S. Tatarenko, Appl. Phys. Lett. 89, 152508 (2006). 